\title{
CONTRIBUIÇÕES BIOECOLÓGICAS PARA COMPREENSÃO DA MOTIVAÇÃO EM MUSCULAÇÃO - ARTIGO DE REVISÃO
}

\author{
Joacks de Paula Lemos Filho' ${ }^{1}$, Renato Henrique Verzani ${ }^{2}$, Kauan Gauvão Morão ${ }^{3}$, \\ Ivan Wallan Tertuliano ${ }^{4}$, Arthur Bernardino Domene Senna ${ }^{5}$, \\ Afonso Antonio Machado ${ }^{6}$
}

Resumo: A motivação é fundamental em diversos contextos, como nas práticas de exercícios e atividades físicas. Com isso, o objetivo deste estudo foi de identificar quais são os aspectos relacionados com a motivação que conduzem a prática de musculação, tendo por base a teoria bioecológica de Urie Bronfenbrenner. Assim realizou-se um estudo exploratório a partir de uma pesquisa bibliográfica, utilizando as bases de dados Scielo e Google acadêmico, buscando trabalhos publicados entre os anos de 2011 e 2016. Esta pesquisa possibilitou constatar que os motivos que foram mais citados dentre os adeptos da musculação foram a estética e a saúde. Diante desses resultados, sob a ótica da teoria bioecológica é viável considerar a musculação como microssistema, que pode sofrer influências dos macrossistemas, tais como a mídia, por exemplo. Portanto, é possível concluir que os macrossistemas exercem influência sobre os processos proximais das pessoas, interferindo no comportamento e desenvolvimento dos mesmos.

Palavras-chave: Desenvolvimento humano. Motivação. Musculação. Teoria bioecológica.

1 Acadêmico do curso de Educação Física da Universidade Estadual Paulista - Unesp, Rio Claro. Membro do Laboratório de Estudos e Pesquisas em Psicologia do Esporte - Lepespe - Unesp, Rio Claro.

2 Mestre, Doutorando em Desenvolvimento Humano e Tecnologias pela Universidade Estadual Paulista - Unesp, Rio Claro. Membro do Laboratório de Estudos e Pesquisas em Psicologia do Esporte Lepespe - Unesp, Rio Claro.

3 Mestre, Doutorando em Desenvolvimento Humano e Tecnologias pela Universidade Estadual Paulista - Unesp, Rio Claro. Membro do Laboratório de Estudos e Pesquisas em Psicologia do Esporte Lepespe - Unesp, Rio Claro.

4 Doutor, Docente do Centro Universitário Adventista de São Paulo - Unasp, São Paulo. Membro do Laboratório de Estudos e Pesquisas em Psicologia do Esporte - Lepespe - Unesp, Rio Claro.

5 Psicólogo, Mestrando em Desenvolvimento Humano e Tecnologias pela Universidade Estadual Paulista - Unesp, Rio Claro. Membro do Laboratório de Estudos e Pesquisas em Psicologia do Esporte - Lepespe - Unesp, Rio Claro.

6 Doutor, Docente na Universidade Estadual Paulista - Unesp, Rio Claro. Membro do Laboratório de Estudos e Pesquisas em Psicologia do Esporte - Lepespe - Unesp, Rio Claro. 


\title{
BIOECOLOGICAL CONTRIBUTIONS FOR UNDERSTANDING MOTIVATION IN BODYBUILDING - REVIEW ARTICLE
}

\begin{abstract}
Motivation is critical in many contexts, such as exercise and physical activity practices. Thus, the objective of this study was to identify the aspects related to the motivation that lead to the practice of bodybuilding, based on the bioecological theory of Urie Bronfenbrenner. An exploratory study was carried out based on a bibliographical research, using Scielo and Google academic databases, searching for works published between the years 2011 and 2016. This research made it possible to verify that the motives that were most cited among the followers of the Bodybuilding were aesthetics and health. In view of these results, from the point of view of bioecological theory it is feasible to consider bodybuilding as a microsystem, which can be influenced by macrosystems, such as the media, for example. Therefore, it is possible to conclude that macrosystems influence the proximal processes of people, interfering in the behavior and development of the same.
\end{abstract}

Keywords: Human development. Motivation. Bodybuilding. Bioecological theory.

\section{INTRODUÇÃO}

Diversos são os desafios que envolvem a prática esportiva e a atuação profissional, necessitando assim de novos olhares e atitudes que visem colaborar da melhor maneira possível para superá-los. Assim, surgem teorias que colaboram com a superação desta demanda, como no caso do modelo bioecológico, que de acordo com Krebs et al. (2008), ainda tem sua utilização no contexto esportivo de modo muito incipiente.

Uma das principais teorias é a Bioecológica de Urie Bronfenbenner, na qual o desenvolvimento de uma pessoa é compreendido pela relação de quatro elementos, sendo eles o processo, a pessoa, o contexto e o tempo (FONTES; BRANDÃO, 2013). Desta maneira, o progresso das pessoas na sociedade estaria diretamente envolvido com estes quatro elementos norteadores.

Caminhando neste sentido, o progresso estaria envolvido com o resultado da relação e interação entre a pessoa e o contexto. Com isso, a vivência dos indivíduos no meio desencadeia alterações bilaterais, facilitando o desenvolvimento. Este envolvimento tem relação direta com as características da pessoa, isto é, fatores genéticos, psicológicos e biológicos.

O contexto em que a pessoa se encontra possui uma subdivisão em níveis, sendo estes o microssistema, mesossistema, exossistema e macrossistema. Sendo assim, há uma relação de proximidade em que o ambiente poderia ser comparado às bonecas russas, que vão se encaixando da maior para a menor, podendo a maior ser comparada com o macrossistema e a menor com o microssistema, movendo-se do nível interno até o externo (BENETTI et al., 2013). Há uma influência cronológica nestes elementos, ou seja, o tempo, os eventos históricos e os períodos estão relacionados aos elementos do constructo. 
A escola, a família e outros tipos de interações mais diretas formam o que é chamado de microssistema (YUNES, 2010). Assim, temos que a musculação poderia ser entendida como um envolvimento social que compõe o micro contexto, criando uma interferência recíproca. A musculação, que para Liz (2011) está relacionada com exercícios físicos envolvendo sobrecargas, realizadas de diversas maneiras, como em máquinas desenvolvidas para esta finalidade, elásticos, halteres, dentre outros, faz parte das atividades desenvolvidas pela humanidade.

A prática esportiva por meio deste tipo de treinamento faz parte do desenvolvimento humano, pois há uma interação entre o ambiente e o indivíduo. Com isso, as dimensões da teoria bioecológica podem ter relação com questões motivacionais, tais como adesão, continuidade ou mesmo desistência da prática de musculação.

Este cenário tem atraído números cada vez maiores de adeptos, com as mais diversas finalidades, podendo ser estética, saúde, aumento da força muscular, entre outros. Sendo assim, de acordo com Domenico (2009), há benefícios na prevenção, tratamento e recuperação, o que pode ser importante em qualquer faixa etária, inclusive na terceira idade, melhorando a funcionalidade no dia-a-dia e podendo ajudar em diversos outros aspectos relacionados com o processo de envelhecimento.

Quanto a motivação, Pina et al. (2014) destacam que existem propriedades multicausais, podendo ser influenciadas de modo intrínseco, isto é, limitações ou mesmo a personalidade do sujeito e também extrínseco, no caso da cultura, do contexto, do tempo, dentre outros. A motivação intrínseca possui relação direta com o fato de uma pessoa realizar certa atividade por uma satisfação inerente a esta (RYAN; DECI, 2000). Isto é, as pessoas buscam prazer para si próprias, o que Balbinotti (2008) destaca que acaba por conduzir a práticas ou atividades por vontade própria, além do prazer, etc.

$\mathrm{Na}$ motivação extrínseca, por sua vez, Ryan e Deci (2000) salientam que a realização da tarefa ou atividade busca conseguir atingir determinado objetivo não apenas pela própria satisfação, o que Amorim (2010) destaca como sendo algo que vai além desta, isto é, recompensas, reconhecimento, prêmios, dentre outros. Não podemos deixar de citar também a amotivação, que é a inexistência de vontade e proatividade. Vallerand (1992) afirma que o comportamento desmotivado não é nem extrínseco e nem intrínseco, isto é, não conta com motivação.

A teoria da autodeterminação baseia-se na motivação e a relação desta com o desenvolvimento humano, levando em consideração a autonomia motivacional, a motivação controlada e também a amotivação. Estes três componentes geram influência direta no desempenho, no bem-estar e nas relações (DECI; RYAN, 2008).

É importante deixar claro que, por meio da teoria bioecológica, o desenvolvimento humano é algo continuo, no qual faz parte a existência de alterações e adaptações do ponto de vista biopsicológico, temos que tudo que passou e também o presente geram influências (BRONFENBRENNER, 2012). 
Diante de todo exposto, o objetivo do presente estudo foi utilizar a teoria Bioecológica de Bronfenbrenner para compreensão da motivação na prática de musculação.

\section{PROCEDIMENTOS METODOLÓGICOS}

A pesquisa foi realizada por meio de revisão bibliográfica, utilizando as plataformas Google Acadêmico e Scielo na busca e, além disso, com foco apenas nos artigos em língua portuguesa. As buscas foram realizadas entre os meses de agosto e outubro de 2016 utilizando os seguintes descritores: "motivação em praticantes de musculação", "motivação e treinamento resistido", "motivos e musculação" e "motivação e musculação". Foram excluídos trabalhos que compreendiam participantes de idade abaixo dos 18 anos, já que se trata de motivação para prática de musculação, assim como trabalhos de revisão e publicados antes de 2011.

A busca inicial totalizou 23 trabalhos dos quais 07 foram excluídos por duplicata. Dos 16 trabalhos restantes para leitura, 4 foram excluídos por não preencherem ao critério de pesquisa de campo, restando 12 trabalhos, os quais foram lidos na íntegra e fizeram parte dos resultados deste estudo por apresentarem informações relevantes. Desse 12 trabalhos, 8 são artigos nacionais e 4 são monografias.

\section{RESULTADOS E DISCUSSÃO}

A partir do desenho metodológico proposto, foram selecionados arquivos diretamente relacionados com o contexto da musculação e também com a análise de aspectos motivacionais desta prática, que é representativa atualmente e busca seguir na contramão do estilo de vida contemporâneo, no qual diversas novas tecnologias têm afastado as pessoas da prática de atividades físicas, colocando-as em estilos de vida sedentários.

Estar atento aos fatores que influenciam os hábitos da população é uma necessidade. Freitas et al. (2007) salientam que a busca por melhora na qualidade de vida e também na saúde a partir de práticas corporais ou atividades físicas é algo que possui relação direta com questões complexas e diversificadas nesta sociedade globalizada.

Com o intuito de verificar a motivação para prática de atividade física, Lima (2012) aplicou o inventário de motivação à prática de atividade física (IMPRAF-54). Seus resultados demonstraram que a saúde é o principal motivo para prática e adesão da musculação e que a estética corporal aparece como o segundo maior motivo para prática. Souza (2012), Moraes (2015) e Moreira (2015), realizaram uma pesquisa por meio de questionários adaptados, com o intuito de compreender os componentes motivacionais de praticantes de musculação. Como resultado os estudos citados encontraram que o 
aspecto da saúde é o principal fator motivacional para a prática do treinamento resistido. Outro fator importante apontado nas pesquisas é a estética corporal.

O recente estudo de Liz (2016), construído por meio de uma entrevista semiestruturada, também possibilita a compreensão da saúde e estética como fatores motivacionais identificados na adesão desta prática. Assim, estes estudos demonstram que além da saúde, a estética possui um papel importante na motivação dos indivíduos que frequentam academias de musculação. Este comportamento motivacional é fruto de uma indústria corporal que reforça padrões e imagens corporais ideais. (RUSSO, 2005). Ou seja, o "culto ao corpo" ideal direciona a sociedade a buscar e manter algum tipo de atividade física (TAHARA, 2003).

Diante dos resultados apontados anteriormente, e utilizando-se da teoria bioecológica de Bronfenbrenner para compreensão dos achados, pode-se analisar que a busca pela melhora ou manutenção da saúde compreende ao fenômeno sociocultural que permeia a prática de exercícios físicos. Ou seja, a relação entre exercício físico e saúde é transmitida para os diversos contextos sociais.

Estes ambientes fomentam, por meio dos processos proximais, o desenvolvimento humano e, por conseguinte, a motivação de um indivíduo para uma determinada situação. Para Dessen (2007), os processos proximais são fundamentais para promover ou inibir o desenvolvimento, seja ele social, físico ou mental, pois fazem parte dos contextos mais próximos, como no caso da escola ou da família, alterando assim o comportamento das pessoas.

Os contextos mais próximos são influenciados diretamente pelas demais camadas sistêmicas. Desta forma, ao analisarmos as vias midiáticas como contextos macrossistêmicos, é possível identificar suas influências na sociedade e nos demais âmbitos. A prática de exercício físico e mais precisamente a musculação, são afetadas pelo contexto midiático que aponta os benefícios, vantagens e riscos da prática.

Neste sentido, Tahara (2003) aponta que estes canais midiáticos podem gerar influências no sentido de direcionar as pessoas para academia ou práticas esportivas, inclusive pela divulgação de hábitos de vida saudáveis. Assim, visto como um macrossistema, a mídia influencia por meio de seus conteúdos os contextos menores, como o comportamento humano, a adesão a musculação e a melhora da saúde.

De acordo com Amorim (2010), esta possui grande influência na sociedade ao pressioná-la a um padrão de corpo e beleza. Ao analisarmos está questão por meio da perspectiva bioecológica, é possível identificar a relação bidirecional e indissociável apontada por Bronfenbrenner (2012), entre contextos mais distantes (macro) e os proximais (micro). Isto é, o desenvolvimento humano e consequentemente seus engajamentos são alimentados pelos demais contextos.

Neste sentido, a indústria corporal e a mídia, como contextos macrossistêmicos, influenciam os processos proximais e o comportamento das pessoas. Portanto, "a 
motivação destas pessoas encontra-se no desejo de sentirem-se bonitas, desejadas, e veem na atividade física um meio de conseguir o que querem, com relação a si e aos outros no ambiente que a cerca" (LIMA, 2012, p 32).

A influência da estética nos praticantes de musculação também pode ser observada nos estudos de Beppu (2011), Pick (2012), Comandulli (2014) e Rodrigues (2016), no qual os resultados apontam a estética corporal como o principal fator motivacional na adesão ao treinamento resistido, como também na permanência da prática.

As pesquisas acima foram realizadas por meio de questionários e inventários direcionados para análise motivacional. Vale ressaltar que os participantes dos estudos eram compostos por homens e mulheres. Ou seja, a relevância proporcionada pela aparência era mais direcionada as mulheres, porém atualmente também faz parte do contexto do homem contemporâneo (RODRIGUES, 2016). Desta forma, a estética possui um cunho social determinante na motivação extrínseca humana, orientando as características psicológicas essenciais no processo de autoconhecimento e na afirmação do seu espaço na sociedade contemporânea (LIMA, 2012).

Alguns autores como Liz et al. (2013), Barros et al. (2015) e Silva (2016), ao analisarem os fatores motivacionais na prática de musculação, obtiveram como resultados motivações relacionadas a busca de um condicionamento físico, ganho de força e hipertrofia, respectivamente. Estes fatores estão diretamente ligados a melhora da saúde e a busca do corpo ideal. De acordo com Silva, et al. (2006), a força muscular e a manutenção da massa muscular são extremamente relevantes para as capacidades funcionais dos indivíduos.

Em síntese, a maior parte dos resultados apontam para "saúde" e "estética", como fatores motivacionais para prática de musculação (BEPPU, 2011; PICK, 2012; SOUZA, 2012; COMANDULLI, 2014; BARROS et al., 2015; MOREIRA, 2015; MORAES, 2015; LIZ, 2016; RODRIGUES, 2016) , como pode ser observado no Quadro 1.

Quadro 1. Quadro sinóptico dos artigos que versam sobre os fatores motivacionais associados a prática da musculação

\begin{tabular}{|c|c|c|c|c|c|}
\hline ANO & REFERÊNCIA & $\begin{array}{l}\text { PARTICIPAN- } \\
\text { TES (N, Sexo, } \\
\text { Faixa Etária) }\end{array}$ & $\begin{array}{l}\text { TIPO DE } \\
\text { ESTUDO }\end{array}$ & $\begin{array}{l}\text { METODOLO- } \\
\text { GIA }\end{array}$ & $\begin{array}{l}\text { PRINCIPAIS } \\
\text { FATORES MO- } \\
\text { TIVACIONAIS }\end{array}$ \\
\hline 2011 & Beppu & $\begin{array}{c}35 \mathrm{M} \\
21-50 \text { Anos }\end{array}$ & $\mathrm{D}-\mathrm{QL}$ & $\begin{array}{l}\text { Questionário } \\
\text { Adaptado }\end{array}$ & $\begin{array}{c}\text { Estética, redução } \\
\text { massa gorda }\end{array}$ \\
\hline 2012 & Souza & $\begin{array}{c}91(70 \mathrm{M} / 21 \mathrm{H}) \\
18-64 \text { Anos }\end{array}$ & LO - QT & $\begin{array}{l}\text { Questionário } \\
\text { adaptado }\end{array}$ & Saúde, estética \\
\hline 2012 & Pick & $\begin{array}{c}33(21 \mathrm{M} / 12 \mathrm{H}) \\
16-50 \text { Anos }\end{array}$ & D - QT & $\begin{array}{l}\text { Questionário } \\
\text { Adaptado }\end{array}$ & $\begin{array}{c}\text { Estética, } \\
\text { condicionamento } \\
\text { físico }\end{array}$ \\
\hline 2012 & Lima & $\begin{array}{c}47(33 \mathrm{M} / 14 \mathrm{H}) \\
18-40 \text { Anos }\end{array}$ & $\begin{array}{c}\mathrm{D}-\mathrm{QT} / \mathrm{QL} \\
-\mathrm{T}\end{array}$ & IMPRAF-54 & Saúde, estética \\
\hline
\end{tabular}




\begin{tabular}{|c|c|c|c|c|c|}
\hline ANO & REFERÊNCIA & $\begin{array}{l}\text { PARTICIPAN- } \\
\text { TES (N, Sexo, } \\
\text { Faixa Etária) }\end{array}$ & $\begin{array}{l}\text { TIPO DE } \\
\text { ESTUDO }\end{array}$ & $\begin{array}{l}\text { METODOLO- } \\
\text { GIA }\end{array}$ & $\begin{array}{c}\text { PRINCIPAIS } \\
\text { FATORES MO- } \\
\text { TIVACIONAIS }\end{array}$ \\
\hline 2013 & Liz et al. & \begin{tabular}{|c|}
$86(43 \mathrm{M} / 43 \mathrm{H})$ \\
Média 29 Anos M, \\
26 Anos H
\end{tabular} & $\mathrm{D}-\mathrm{QL}$ & BREQ-2 & $\begin{array}{l}\text { Condicionamento } \\
\text { físico, estética }\end{array}$ \\
\hline 2014 & Comandulli & $\begin{array}{c}100 \mathrm{H} \\
20-40 \text { Anos }\end{array}$ & $\mathrm{D}-\mathrm{QT}$ & IMPRAF-54 & Estética, saúde \\
\hline 2015 & Moreira & $\begin{array}{c}150(78 \mathrm{M} / 72 \mathrm{H}) \\
18-68 \text { Anos }\end{array}$ & $D-E$ & $\begin{array}{l}\text { Questionário } \\
\text { adaptado }\end{array}$ & Saúde, estética \\
\hline 2015 & Moraes & $\begin{array}{c}80(45 \mathrm{M} / 35 \mathrm{H}) \\
\text { A partir de } 25 \text { Anos }\end{array}$ & $\mathrm{T}-\mathrm{E}$ & $\begin{array}{l}\text { Questionário } \\
\text { adaptado }\end{array}$ & Saúde, estética \\
\hline 2015 & Barros et al. & $\begin{array}{c}35(19 \mathrm{M} / 16 \mathrm{H}) \\
18-30 \text { Anos }\end{array}$ & $\mathrm{D}-\mathrm{QL}-\mathrm{T}$ & $\begin{array}{l}\text { Questionário } \\
\text { semiestruturado }\end{array}$ & $\begin{array}{c}\text { Ganho de força/ } \\
\text { qualidade de vida, } \\
\text { estética }\end{array}$ \\
\hline 2016 & $\mathrm{Liz}$ & $\begin{array}{l}13(6 \mathrm{M} / 7 \mathrm{H}) \\
23-55 \text { Anos }\end{array}$ & $\mathrm{D}-\mathrm{QL}$ & $\begin{array}{c}\text { Entrevista } \\
\text { semiestruturada }\end{array}$ & $\begin{array}{c}\text { Bem-estar, saúde, } \\
\text { estética }\end{array}$ \\
\hline 2016 & Rodrigues & $\begin{array}{c}35(\mathrm{M} / \mathrm{H}) \\
18-25 \text { Anos }\end{array}$ & $\mathrm{D}-\mathrm{QT} / \mathrm{QL}$ & $\begin{array}{l}\text { Questionário } \\
\text { Misto }\end{array}$ & Estética, saúde \\
\hline 2016 & Silva & \begin{tabular}{|c|}
$30(15 \mathrm{M} / 15 \mathrm{H})$ \\
Média $=25,16$ anos
\end{tabular} & $\mathrm{D}-\mathrm{QT}-\mathrm{T}$ & $\begin{array}{l}\text { Questionário } \\
\text { adaptado }\end{array}$ & $\begin{array}{c}\text { Hipertrofia, } \\
\text { emagrecimento }\end{array}$ \\
\hline
\end{tabular}

Nota: Abreviações presentes no quadro: D, descritiva; T, transversal; E, exploratória; QT, quantitativa; QL, qualitativa; LO, levantamento de opinião; M, mulheres; H, homens; IMPRAF-54, inventário de motivação a prática regular de atividade física; BREQ-2, Questionário de Regulação de Comportamento no Exercício Físico.

Portanto, a motivação para prática de musculação possui uma relação entre questões intrínsecas e extrínsecas da pessoa, dependendo assim das inter-relações dinâmicas envolvendo os processos proximais e os inúmeros contextos que podem ser influenciadores do bem-estar biopsicossocial, como apontado por Benetti (2013).

\section{CONSIDERAÇÕES FINAIS}

A prática de musculação tem conquistado espaço e, por meio de pesquisas, cada vez encontramos mais especificidades e conhecemos novos benefícios relacionados a mesma. Refletindo sobre os processos que surgem a partir da interação de micros contextos, podemos considerar este como um ambiente que torna viável a interação com os indivíduos, podendo esta evoluir com o tempo e alterar processos proximais e a motivação humana.

Diante dos resultados apresentados, a musculação é um microssistema e que sobre influências de outros níveis, isto é, é modelada de acordo com os outros ambientes mais externos (como a cultura, por exemplo), podendo influenciar a motivação dos praticantes. Considerando então que os fatores motivacionais mais apontados nesta pesquisa foram saúde e estética, pode-se considerar que os mesmos estão intimamente relacionados com o macrossistema da sociedade contemporânea, reforçando a influência 
dos contextos mais externos e da importância do modelo bioecológico no entendimento da dinâmica motivacional relacionada à prática.

\section{REFERÊNCIAS}

BALBINOTTI, M. A. A. Motivação à prática regular de atividade física: um estudo exploratório com praticantes em academias de ginástica. Revista Brasileira de Educação Física e Esporte, São Paulo, v. 22, n. 1, p. 63-80, 2008.

BARBOSA, R, R. M. Treinamento resistido; estética e saúde: uma revisão de literatura. 2011. 20 f. Trabalho de conclusão de curso (licenciatura plena em educação física) Universidade do Estado do Pará, Belém, 2011.

BARROS, G. R; CASTELLANO, S. M; SILVA, S. F; DE PAULO, T. R. S. Motivos para a prática de musculação entre adultos Jovens de uma academia no município de Parintins/am. Acta Brasileira do Movimento Humano, v. 5, n. 1, p.66-75, 2015.

BENETTI, C. I; VIEIRA, M. L; CREPALDI, M. A; SCHNEIDER, D. R. Fundamentos da teoria bioecológica de Urie Bronfenbrenner. Pensando Psicología, v.9, n. 16, p. 89-99, 2013.

BEPPU, S. R. G. Motivos que levam as pessoas a frequentarem a academia de musculação em busca do corpo belo. Encontro Internacional de Produção Científica Cesumar, n. 7, 2011, Maringá. Anais... Maringá, Cesumar, p.1-4. 2011.

BRONFENBENNER, U. Bioecologia do Desenvolvimento Humano: tornando os seres humanos mais humanos. 1. ed. Porto Alegre: Artmed, 2012.

COMANDULLI, D. M. N. Fatores motivacionais relacionados à prática do treinamento resistido na cidade de Curitiba/PR. 2014. 51 f. Trabalho de conclusão de curso (Bacharelado Educação Física) - Universidade Tecnológica Federal do Paraná, Curitiba, 2014.

DECI, E. L; RYAN, R.M. Self-Determination Theory: A Macrotheory of Human Motivation, Development, and Health. Canadian Psychology, v. 49, n. 3, p.182-185, 2008.

DESSEN, M. A. A Família e a Escola como contextos de desenvolvimento humano. Revista Paidéia, Ribeirão Preto, v. 17, n. 36, p 21-32, 2007.

DOMENICO, L. D. Motivação em idosos praticantes de musculação. Lecturas: Educación Física y Deportes, Bueno Aires, v. 130, n. 13, p.01-13, 2009.

FONTES, R. C. C; BRANDÃO, M. R. F. A resiliência no âmbito esportivo: uma perspectiva bioecológica do desenvolvimento humano. Motriz, Rio Claro, v. 19, n. 1, p.151159, 2013.

FREITAS, C. M. S. M; SANTIAGO, M. S; VIANA, A. T; LEÃO, A. C; FREYRE, C. Aspectos motivacionais que influenciam a adesão e manutenção de idosos a programas de exercícios físicos. Revista Brasileira de Cineantropometria e Desempenho Humano, v. 9, n. 1, p. 92-100, 2007. 
KREBS, R. J; COPETTI, F; SERPA, S; ARAUJO, D. Revista Brasileira de Psicologia do Esporte, São Paulo, v. 2, n. 2, p.1-24, 2008.

LIMA, R. S. Análise de fatores motivacionais em iniciantes à prática de musculação em academia. 2012. 57 f. Trabalho de conclusão do curso (Bacharel em Educação Física) Universidade Federal de Santa Catarina, Florianópolis, 2012.

LIZ, C. M, Análise qualitativa dos motivos de adesão e desistência da musculação em academias. Revista Brasileira de Ciência do Esporte, v. 38, n. 3, p. 267-274. 2016.

LIZ, C. M. Motivação para a prática de musculação de aderentes e desistentes de academias Florianópolis. 2011. 245 f. Dissertação (Mestrado em Ciências do Movimento Humano) - Universidade do Estado de Santa Catarina, Florianópolis, 2011.

LIZ, C. M; VIANA, M. S; BRANDT, R; LAGOS, N. R; VASCONCELLOS, D. I.C; ANDRADE, A. Aspectos motivacionais para a prática de exercício resistido em academias. Educação Física em Revista, v. 7, n. 1, p.1-14. 2013.

MORAES, L. M. Aspectos motivacionais para a prática de exercícios em academias, 2015. 27 f. Trabalho de conclusão de curso. (Bacharel em Educação Física) - Faculdade de Ciências da Educação e Saúde, Brasília, 2015.

MOREIRA, J. A. Motivos que levam as pessoas a praticarem exercícios em uma academia de musculação: um estudo de caso. 2015. 10 f. Trabalho de conclusão de curso. (Bacharel em Educação Física) - Universidade do Extremo Sul Catarinense, Criciúma, 2015.

PICK, F. J. Motivação em academias através de redes sociais. 2012. 38 f. Trabalho de conclusão de curso. (Bacharel em Educação Física) - Universidade Federal de Santa Catarina, Florianópolis, 2012.

PINA, F. L. C; DANTAS, J. L; CAMPOS FILHO, M. G. A; LIDO, D. J; CONTI, P. S. Motivação para a prática de exercícios com pesos: influência da supervisão e do gênero. Revista Brasileira de Atividade Física e Saúde, Pelotas, v. 2, n. 19, p.168-177, 2014.

RODRIGUES, A. L. P. Aspectos motivacionais para a prática de musculação entre jovens de 18 a 25 anos do município de Fortaleza-CE. Revista Brasileira de Prescrição e Fisiologia do Exercício, v. 10, n. 38, p. 308-313, 2016.

RYAN, R. M; DECI, E. L. Intrinsic and Extrinsic Motivations: Classic Definitions and New Directions. Contemporary Educational Psychology, v. 25, n. 1, p.54-67, 2000.

SILVA, A, J. B. Motivações dos praticantes de musculação de uma academia em Canindé-ce. Revista ENAF Science, v. 11, n. 1, p.196-205, 2016.

SILVA, C. M.; GURJÃO, A. L. D.; FERREIRA, L.; GOBBI, L.T.B.; GOBBI, S. Efeito do treinamento com pesos, prescrito por zona de repetições máximas, na força muscular e composição corporal em idosas. Revista Brasileira de Cineantropometria \& Desempenho Humano, Santa Catarina, v. 4, n. 8, p.39-45, 2006.

SOUZA, J. C. C. Percepções e motivações acerca da prática de musculação de uma academia de ginástica da baixada fluminense. Revista Corpus et Scientia, Rio de Janeiro, v. 8, n. 2, p. 66-77, 2012. 
TAHARA, A. K. Aderência e manutenção da prática de exercícios em academias. Revista Brasileira de ciência e movimento, Brasília, v. 11, n. 4, p.7-12, 2003.

YUNES, M. A. M; JULIANO, M. C. A Bioecologia do Desenvolvimento Humano: Conceitos fundamentais e possibilidades de interlocução com a Educação Ambiental.

Cadernos de Educação, v. 37, p. 347-379, 2010. 